\title{
Perfusion imaging with arterial spin labeling (ASL)-MRI predicts malignant progression in low-grade (WHO grade II) gliomas
}

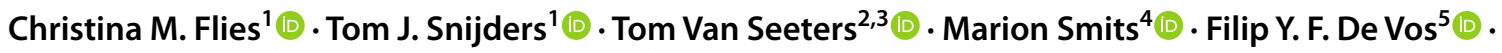 \\ Jeroen Hendrikse $^{2}$ [D . Jan Willem Dankbaar ${ }^{2}$ (I)
}

Received: 5 March 2021 / Accepted: 23 May 2021 / Published online: 11 June 2021

(c) The Author(s) 2021

\begin{abstract}
Purpose Predicting malignant progression of grade II gliomas would allow for earlier initiation of treatment. The hypothesis for this single-centre, case-control study was that the perfusion signal on ASL-MRI predicts such malignant progression in the following 12 months.

Methods Consecutive patients with the following criteria were included: $\geq 18$ years, grade II glioma (biopsied or resected) and an ASL-MRI 6-12 months prior to malignant progression (cases) or stable disease (controls). Malignant progression was defined either radiologically (new T1w-contrast enhancement) or histologically (neurosurgical tissue sampling). Three controls were matched with each case. Some patients served as their own control by using earlier imaging. The ASL-MRIs were reviewed by two neuroradiologists and classified as positive (hyper-intense or iso-intense compared to cortical grey matter) or negative (hypo-intense). In patients with epilepsy, a neurologist reviewed clinicoradiological data to exclude periictal pseudoprogression. The statistical analysis included diagnostic test properties, a Cohen's Kappa interrater reliability coefficient and stratification for previous radiotherapy.

Results Eleven cases (median age $=48, \mathrm{IQR}=43-50$ years) and 33 controls $(43,27-50$ years) were included. Malignant progression appeared at 37 months (median, IQR $=17-44$ ) after first surgery. Thirty ASL-MRIs were assessed as negative and 14 as positive. None of the MRIs showed signs of peri-ictal pseudoprogression. ASL significantly predicted subsequent malignant progression (sensitivity $=73 \%$; specificity $=82 \%$; OR $=12 ; 95 \%-\mathrm{CI}=2.4-59.1 ; \mathrm{p}=0.002$ ). The interrater reliability coefficient was 0.65 . In stratified analysis, ASL-MRI predicted malignant progression both in patients with previous radiotherapy and in those without (Mantel-Haenszel test, $\mathrm{p}=0.003$ ).

Conclusion Perfusion imaging with ASL-MRI can predict malignant progression within 12 months in patients with grade II glioma.
\end{abstract}

Keywords Arterial spin labeling $\cdot$ Low-grade glioma $\cdot$ Malignant progression $\cdot$ Perfusion MRI

Tom J. Snijders

T.J.Snijders@umcutrecht.nl

1 Department of Neurology \& Neurosurgery, UMC Utrecht Brain Center, University Medical Center Utrecht, Heidelberglaan 100, 3584 CX Utrecht, the Netherlands

2 Department of Radiology, University Medical Center Utrecht, Utrecht, the Netherlands

3 Department of Radiology, Elisabeth-TweeSteden Ziekenhuis, Tilburg, the Netherlands

4 Department of Radiology and Nuclear Medicine, Erasmus MC, University Medical Centre Rotterdam, Rotterdam, the Netherlands

5 Department of Medical Oncology, University Medical Center Utrecht, Utrecht, the Netherlands

$\begin{array}{ll}\text { Abbreviations } \\ \text { ASL } & \text { Arterial spin labeling } \\ \text { MP } & \text { Malignant progression } \\ \text { LGG } & \text { Low-grade glioma } \\ \text { RT } & \text { Radiotherapy } \\ \text { IDH } & \text { Isocitrate dehydrogenase } \\ \text { WHO } & \text { World Health Organisation } \\ \text { CE } & \text { Contrast enhancement }\end{array}$

\section{Introduction}

Diffuse gliomas are the most common adult-onset primary malignant brain tumours [1] and are classified in the World Health Organisation (WHO) 2016 criteria 
according to histological and molecular-genetic characteristics as grade II, III or IV [2]. Low-grade gliomas (LGGs, WHO grade II) include isocitrate dehydrogenase (IDH)-mutant astrocytomas, IDH-mutant and $1 \mathrm{p} / 19 \mathrm{q}-$ codeleted oligodendrogliomas and the more aggressive IDH-wildtype tumours. Overall survival in IDH-mutant LGGs varies from 5 to more than 13 years, depending on patient characteristics, histomolecular subtypes and treatment forms [3, 4].

The duration of survival is largely determined by the occurrence of dedifferentiation, or 'malignant progression' (MP). MP of grade II into grade III or IV gliomas inevitably follows after a variable period of time [4]. Predicting MP would permit for earlier initiation of therapy. MP was described in 44 of 216 patients with LGG after a median time of 10.1 years (range for the patients treated with radiotherapy $=19-79$ months) and in another observational study, in 124 of 599 patients with LGG after a median time of 4.7 years (range $=1.6-299.7$ months) [5, 6]. Given this variable time to MP, predicting the moment of MP in individual patients is difficult.

To identify MP as soon as possible, the surveillance of patients with stable LGG consists of regular MRI, usually with 6-month intervals, based on international guidelines [7]. New or increasing regions of enhancement, defined by the RANO criteria as an increase of the perpendicular diameter of the lesion compared to the post-operative MRI [8], are the most commonly used features to identify MP. MR perfusion techniques may have the potential for early identification of MP. DSC, which relies on an intravascular contrast agent, is the most widely used method [9]. The primary parameter that is determined with DSC perfusion is relative CBV (rCBV). Using DSC, previous authors have reported an increase in $\mathrm{rCBV}$ in the 12 months before dedifferentiation compared to patients with stable disease [10]. Another MR perfusion method is arterial spin labeling (ASL). This method does not depend on a contrast agent, but on labelled images with magnetised arterial blood protons [11, 12]. These images are subtracted from control images, containing the same static tissue signals, and CBF maps are generated by averaging many control-label pairs. High relative CBF measurements, as measured with ASL, predicted tumour progression in a cohort of paediatric diffuse astrocytomas, especially in combination with ${ }^{18} \mathrm{~F}$-DOPA-PET and ADC [13]. ASL images can also be assessed visually to identify relative areas of hyperperfusion. This method may be easier to use during daily clinical practice than quantification.

Our hypothesis for this single-centre, retrospective case-control study was that hyperperfusion on ASL-MRI in patients with grade II gliomas predicts malignant progression within 12 months.

\section{Methods}

\section{Patient selection}

The institutional Medical Ethical/Biobank Committee approved the use of patient data in the context of an overlapping not yet published study in progress, for which patients had provided written informed consent (protocol no. 16-342/16-340).

For this single-centre, retrospective case-control study, all patients with a WHO grade II glioma from our local database (which started from January 2000) were reviewed in May 2019. Furthermore, patients with a grade III or IV glioma were reviewed to detect secondary malignant tumours after a first surgery in another hospital, because these patients were not included in our local database of patients with a grade II glioma.

The inclusion criteria were (a) aged 18 years or older at first surgery; (b) histologically confirmed WHO grade II glioma according to WHO 2016 criteria (biopsy or resection) [2]; (c) availability of an ASL-MRI (index MRI) performed as part of routine clinical care, from January 2016 until May 2019, and (d) diagnosis of MP for the cases, or stable disease for the controls. MP was defined as new contrast enhancement (CE) on T1w follow-up MRI and/or histological proof of progression to grade III or IV within the subsequent 12 months after the ASL-index MRI, whereas stable disease was defined as no new CE on T1w follow-up MRI and/or a stable grade II lesion at recurrence-resection within the subsequent 12 months after the ASL-index MRI. The presence of MP was determined blinded to the ASL results by a medical researcher (CMF) and a neuro-oncologist (TJS, 11 years experience) based on the radiological, pathological, and multidisciplinary team reports. Based on histomolecular tumour type (including $1 \mathrm{p} / 19 \mathrm{q}$-status and IDH-status), age ( 2 categories: $18-59$ years and 60 years or over), treatment before the ASL and occurrence of epilepsy, three controls were matched with each case. Cases ideally provided their own control with one ASL-MRI followed by 12 months of stable disease and thereafter another one followed by malignant progression with a period of at least 12 months between the two ASL-MRIs. Previous treatment with radiotherapy (RT) was accepted despite the risk of mistaking radiation-induced pseudoprogression for MP. Consequently, only patients with clinicoradiological follow-up of at least 6 months showing further progression and a conclusion of true progression in the multidisciplinary tumour board were included.

\section{MRI protocol}

Pseudocontinuous ASL perfusion MRI is performed in this hospital for the surveillance of LGG on a 3.0 T MRI scanner 
(Ingenia, Ingenia CX, Achieva, Philips Healthcare, Best, the Netherlands) with the following sequence parameters: repetition time/echo time, $4000 / 16 \mathrm{~ms}$; flip angle, $90^{\circ}$; field of view, $240 \times 240 \times 119 \mathrm{~mm}(\mathrm{RL}, \mathrm{AP}, \mathrm{FH})$; section thickness, $7 \mathrm{~mm}$; in-plane voxel size, $3 \times 3 \times 7 \mathrm{~mm}$; number of slices in the acquisition, 17; WFS (pix)/BW (Hz), 8.254/52.6; post labeling delays, $1525 \mathrm{~ms}$; readout type, 2D EPI; background suppression pulses $=$ on; label duration, $1650 \mathrm{~ms}$; no M0-scan performed; and total scan time, 3 min $19.7 \mathrm{~s}$. The parameters for the three-dimensional T1-weighted imaging include repetition time/echo time, 5.3/2.4 ms; flip angle, $10^{\circ}$; field of view, $230 \times 230 \times 160 \mathrm{~mm}(\mathrm{RL}, \mathrm{AP}, \mathrm{FH})$; and gap, $-0.5 \mathrm{~mm}$. Patients either underwent 3.0 or $1.5 \mathrm{~T}$ MRI for their follow-up, based on availability, so the collection of 3.0 T follow-up MRIs (including ASL) represented a randomly selected subset.

\section{Analysis}

The assessment of all randomly ordered ASL-index MRIs was performed independently by two neuroradiologists (JWD: 10 years experience, TvS: 8 years experience), who were blinded to the outcome and the clinical data. The ASL images were visually graded in three groups: 0 as hypointensity, 1 iso-intensity and 2 hyper-intensity, all compared to cortical grey matter on the perfusion-weighted maps. In general, the cortical grey matter in the same hemisphere closest to the glioma was used for this comparison. No other (quantitative) measurements were performed. A crosshair was used on FLAIR images to identify the location of tumour on the ASL-MRI after automatic registration in our PACS.

To reflect clinical practice, one reader's assessment (by JWD) was used for our main analysis of diagnostic value. The ASL results were dichotomised as positive (grade 1 and 2 ) or negative (grade 0 ). As a sensitivity analysis, we repeated calculations with grade 2 scans classified as positive, and grade 0 and 1 scans as negative. The second reading (by TvS) was only used for the interobserver reliability analysis. For this secondary analysis, each ASL-MRI was reviewed by both observers. Given the goal of the second reading, there was no need for a consensus reading, and no consensus reading was performed.

To explore the clinical significance of ASL findings, we registered the interval between the index ASL scan and the next scan. An ASL scan was considered clinically significant when a patient deteriorated clinically before the planned follow-up MRI due to MP. Presumably, the clinical significance of a positive ASL is highest in cases of earlier-thanplanned follow-up, especially with clinical deterioration, since early ASL-based identification of MP might prevent such deteriorations.
To exclude peri-ictal pseudoprogression as the cause of radiological changes, a neuro-oncologist (TJS) reviewed clinicoradiological data of patients with seizure symptoms within 3 months previous to a positive ASL blinded to the outcome and excluded MRIs showing signs such as cortical or subcortical enhancement, cortical swelling and diffusion restriction [14] from further analysis. Furthermore, a medical researcher (CMF) reviewed electronic patient files for radiological and histomolecular tumour characteristics, date of first surgery, date of progression and seizure symptoms blinded to the ASL results.

\section{Statistics}

We performed the calculation of an odds ratio (OR) with 95\%-confidence interval (95\%-CI), sensitivity and specificity for identifying MP. Continuous variables were expressed as median or mean with an interquartile range (IQR) or standard deviation. P-values $<0.05$, calculated with the Fisher exact test, were considered statistically significant. To test the robustness, we used the assessment of the second less experienced reader (TvS) to perform an interrater reliability analysis (Cohen's Kappa coefficient [15]) with the same dichotomised categories as mentioned above (positive versus negative). Since we only included participants with complete information, we did not have missing data. To estimate whether there was an association between ASL and MP independent of whether the patient received previous RT and independent of their IDH-mutational status, the Mantel-Haenszel test was used. No subgroup analysis of cases with histological follow-up $(n=4)$ versus radiological follow-up was performed due to the small sample size. SPSS (Version 25.0, 2017, IBM SPSS Statistics, Armonk, NY:IBM Corp.) was used for the calculations.

\section{Results}

\section{Patient characteristics}

In total, we identified and reviewed the records of 757 consecutive patients, 307 with a WHO grade II glioma and 450 with a grade III or IV, from which 11 cases and 99 controls were identified. After matching, 11 cases and 33 controls were included in this study; for seven cases, an appropriate control scan was available within the same case. Figure 1 shows the patient selection process. Baseline criteria are given in Table 1. Individual data can be seen in Tables 4 (cases) and 5 (controls) including tumour type, treatment before the ASL, seizures before the ASL and ASL score. For the cases, the time from ASL to MP, from first surgery to MP and from MRI showing MP to second surgery are additionally available in the Appendix. Of these 37 participants, 24 
Fig. 1 Flowchart of the patient selection process

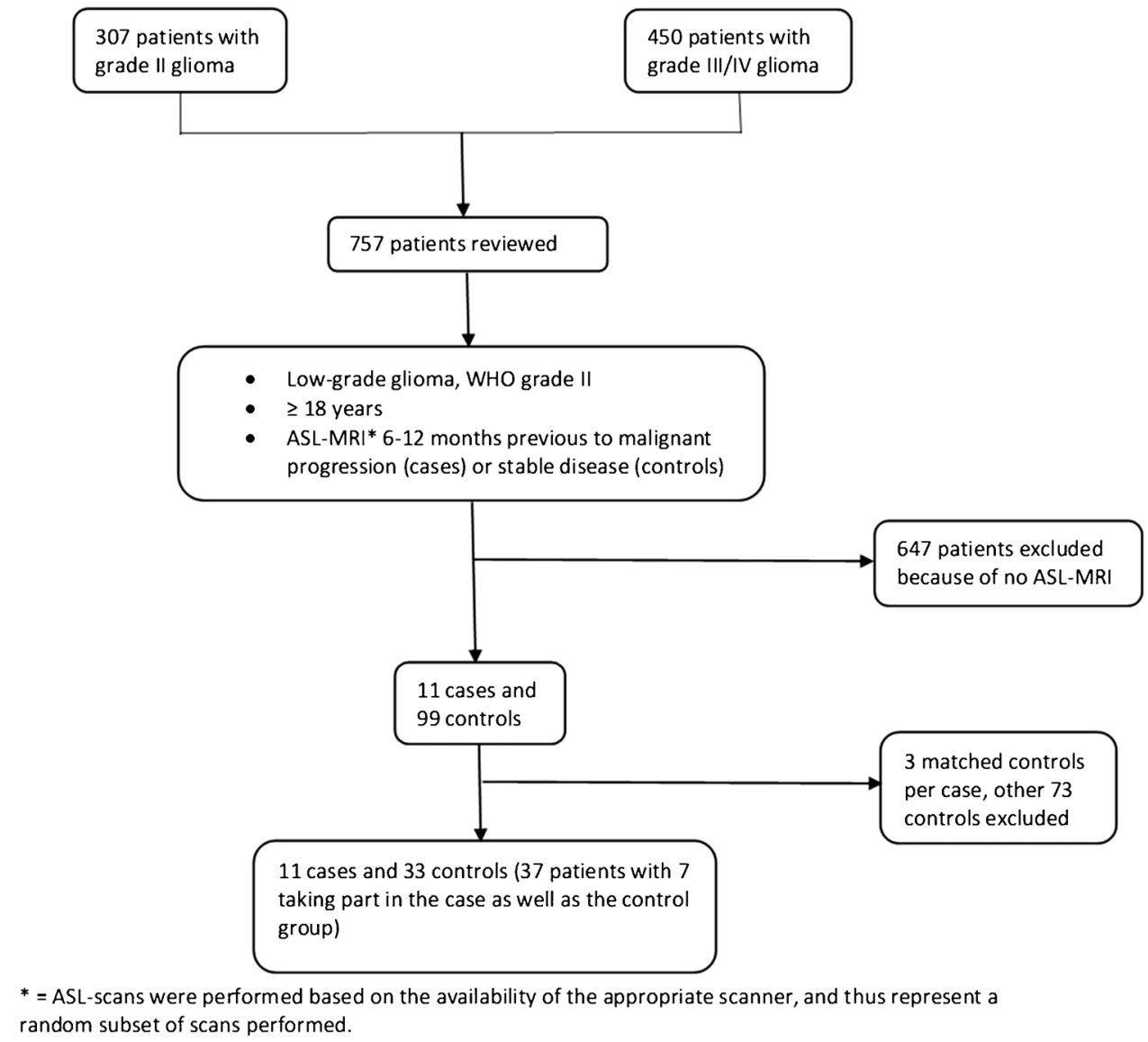

were men and the median age was 45 (IQR 28-50) years with 48 (43-50) years for the cases and 43 (27-50) years for the controls. The majority represented a diffuse astrocytic tumour type. MP occurred at a median of 37 (17-44) months after first surgery. The median interval between the ASL-MRI and the diagnosis of MP was 100 (31-135) days. Treatment before the ASL-MRI included RT and/or temozolomide or procarbazine, lomustine and vincristine chemotherapy. Four cases underwent recurrence resection to confirm the diagnosis, including one without CE on T1wMRI. In the other seven, progression was confirmed radiologically by new T1w-CE. Controls showed persisting stable disease in the next 12 months. Characteristics of the control sample were matched well, though not perfectly, with their matched cases, reflecting the limitations in the availability of controls. Five patients showed an IDH-wildtype glioma (two cases and three matched controls). This diagnosis was based on IDH-sequencing.

\section{Analysis}

We encountered no missing data. Of all 44 ASL-MRIs that were analysed, 14 scans were classified as positive, 5 as 'hyper-intense' compared to cortical grey matter and 9 as 'iso-intense' as seen in Tables 2, 4 and 5. This classification resulted in an OR of 12 (95\%-CI 2.4 to 59.1$)$, a sensitivity of 8 of $11,73 \%$, and a specificity of 27 of $33,82 \%$, with $\mathrm{p}=0.002$. The sensitivity analysis with only hyper-intense scans classified as positive, and iso- or hypo-intense scans as negative, resulted in an OR of 2.22 (95\%-CI 0.3 to 15.4, $\mathrm{p}=0.42$ ). The interrater reliability analysis between the two neuroradiologists produced a kappa value of 0.65 , which represented a substantial agreement. Disagreements between raters were seen in every category (true positive, false positive, true negative, false negative).

When stratifying the results for previous RT, the pooled estimate of the OR was $15(95 \%$-CI 2.6 to $87.7, \mathrm{p}=0.003)$ as seen in Table 2. The stratification for IDH-mutational status produced a common OR of 23.3 (95\%-CI 2.4 to 226.8 , $\mathrm{p}=0.007)$ as seen in Table 3. Typical examples of ASL scans are presented in Figs. 2, 3 and 4.

To exclude interfering effects of epilepsy, six patients, three cases and three controls were reviewed, and none of whom showed signs of peri-ictal pseudoprogression.

\section{Clinical significance of ASL}

Of the eight cases with a positive ASL and eventual MP, five underwent follow-up scanning at an earlier time point than planned. In four cases (cases 1, 2, 6 and 7), the T2w 
Table 1 Baseline criteria

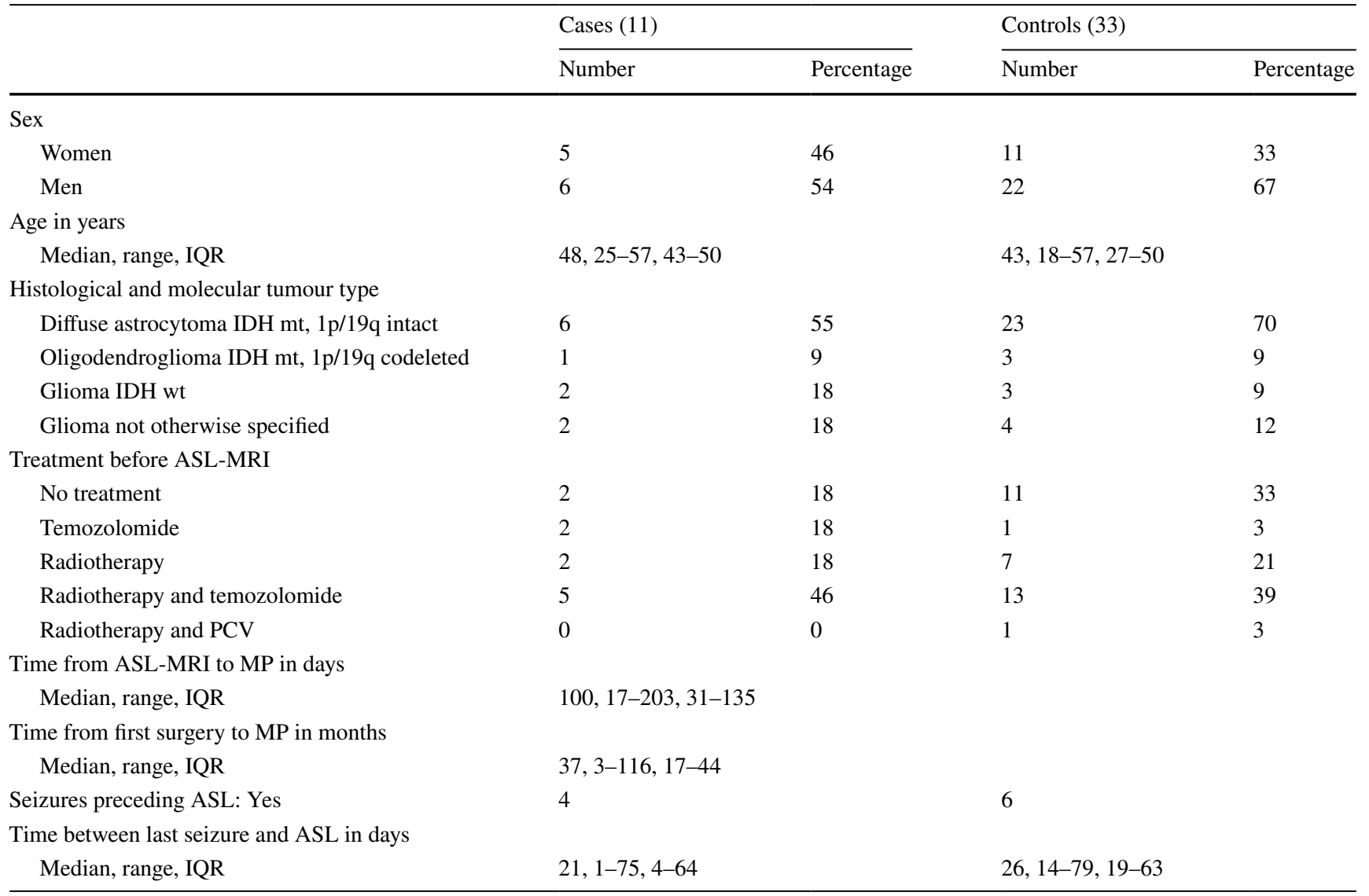

$I Q R$, interquartile range; $I D H w t$, IDH wildtype; $I D H m t$, IDH-mutated; $P C V$, procarbazine/lomustine/vincristine; $M P$, malignant progression; $A S L$, arterial spin labeling MRIs

abnormalities had already been increasing before the evidence of MP, suggesting low-grade progression, and the decision to treat and to shorten the scan interval had already been taken at the time of the index scan. In the fifth case (case 9), an early follow-up scan was performed solely because of clinical deterioration. Of the total number of 14 patients with a positive ASL-MRI ( 8 cases and 6 controls), only the 1 case described above met the requirements of a clinically significant scan and none of the six controls, which represented 13\% (1 of 8) of the positively rated cases. This rate of earlier-than-planned scans was higher in the true positive group (13\%) than the 4\% (1 of 27 ) in the true-negative group.

Table 2 Results 1: RT strata

\begin{tabular}{|c|c|c|c|c|c|c|}
\hline & & & \multicolumn{2}{|c|}{ Malignant progression } & \multirow[t]{2}{*}{$\mathrm{P}$-value } & \multirow{2}{*}{$\begin{array}{l}\text { Com- } \\
\text { mon OR, } \\
95 \% \text {-CI, } \\
\text { p-value }\end{array}$} \\
\hline & & & No & Yes & & \\
\hline \multirow[t]{2}{*}{ No RT } & \multirow[t]{2}{*}{ ASL } & Negative & 9 & 0 & \multirow[t]{2}{*}{0.019} & \\
\hline & & Positive & 3 & 4 & & \\
\hline \multirow[t]{2}{*}{ RT } & \multirow[t]{2}{*}{ ASL } & Negative & 18 & 3 & \multirow[t]{2}{*}{0.043} & \\
\hline & & Positive & 3 & 4 & & \\
\hline \multirow[t]{2}{*}{ Total } & \multirow[t]{2}{*}{ ASL } & Negative & 27 & 3 & \multirow[t]{2}{*}{0.002} & \multirow{2}{*}{$\begin{array}{c}15,2.6- \\
87.7 \\
0.003\end{array}$} \\
\hline & & Positive & 6 & 8 & & \\
\hline
\end{tabular}

$R T$, radiotherapy; $A S L$, arterial spin labeling; $O R$, odds ratio 
Table 3 Results 2: IDH strata

\begin{tabular}{|c|c|c|c|c|c|c|}
\hline & & & \multicolumn{2}{|c|}{ Malignant progression } & \multirow[t]{2}{*}{ P-value } & \multirow{2}{*}{$\begin{array}{l}\text { Com- } \\
\text { mon OR, } \\
95 \% \text {-CI, } \\
\text { p-value }\end{array}$} \\
\hline & & & No & Yes & & \\
\hline \multirow[t]{2}{*}{ IDH wt } & ASL & Negative & 3 & 1 & 0.40 & \\
\hline & & Positive & 0 & 1 & & \\
\hline \multirow[t]{2}{*}{ IDH mt } & ASL & Negative & 20 & 1 & 0.005 & \\
\hline & & Positive & 6 & 6 & & \\
\hline \multirow[t]{2}{*}{ Total } & ASL & Negative & 23 & 2 & 0.003 & $23.3,2.4$ \\
\hline & & Positive & 6 & 7 & & $\begin{array}{l}226.8 \\
0.007\end{array}$ \\
\hline
\end{tabular}

$I D H w t=\mathrm{IDH}$ wildtype $I D H m t=\mathrm{IDH}$-mutated; $A S L=$ arterial spin labeling; $O R=$ odds ratio.

\section{Discussion}

The aim of this study was to investigate the ability of ASL to detect malignant progression (MP) in patients with low-grade glioma (LGG) before MP becomes visible on T1w-MRI. Predicting MP could be of great clinical value, because appropriate treatment adaptations could be started earlier and therefore more effectively. In this way, clinical deterioration might be prevented, resulting in a longer survival time and better quality of life. In this study, a statistically significant association between ASL positivity and MP within 12 months in LGG was noted $(p=0.002)$ with a substantial interrater agreement (Cohen's kappa 0.65). This association persisted after stratification for previous treatment with radiotherapy $(\mathrm{OR}=15, \mathrm{p}=0.003)$ and for IDH-mutational status $(\mathrm{OR}=23.3, \mathrm{p}=0.007)$. Consequently, patients with LGG and a positive ASL should be considered at high risk of impending MP. In these patients, closer follow-up with short imaging intervals could be considered. Currently, in daily clinical practice, ASL positivity alone is not considered sufficient proof of MP.

Previous literature investigating prediction of MP with perfusion MRI is scarce. Danchaivijitr et al. compared the mean value of $\mathrm{rCBV}$ at 18, 12 and 6 months before MP between groups with and without MP and reported an increase in the 12 months before [10]. Morana et al. determined a maximum rCBF with ASL-MRI in combination with ${ }^{18} \mathrm{~F}$-DOPA-PET and ADC for the detection of progression in 26 children with diffuse astrocytoma [13]. PET-scan and diffusion MRI have previously been used to predict MP in LGG. In one study, the interval change of the 10th percentile ADC predicted progression on average 8 months before the RANO criteria in 12 of 13 patients with progressive LGG compared to 3 of 15 with stable disease [16]. In another study, MP appeared after 2 to 27 months after initial diagnosis in $78 \%$ of diffuse LGG with ${ }^{18} \mathrm{~F}$-FETPET uptake shortly after the diagnosis compared to $0 \%$ with

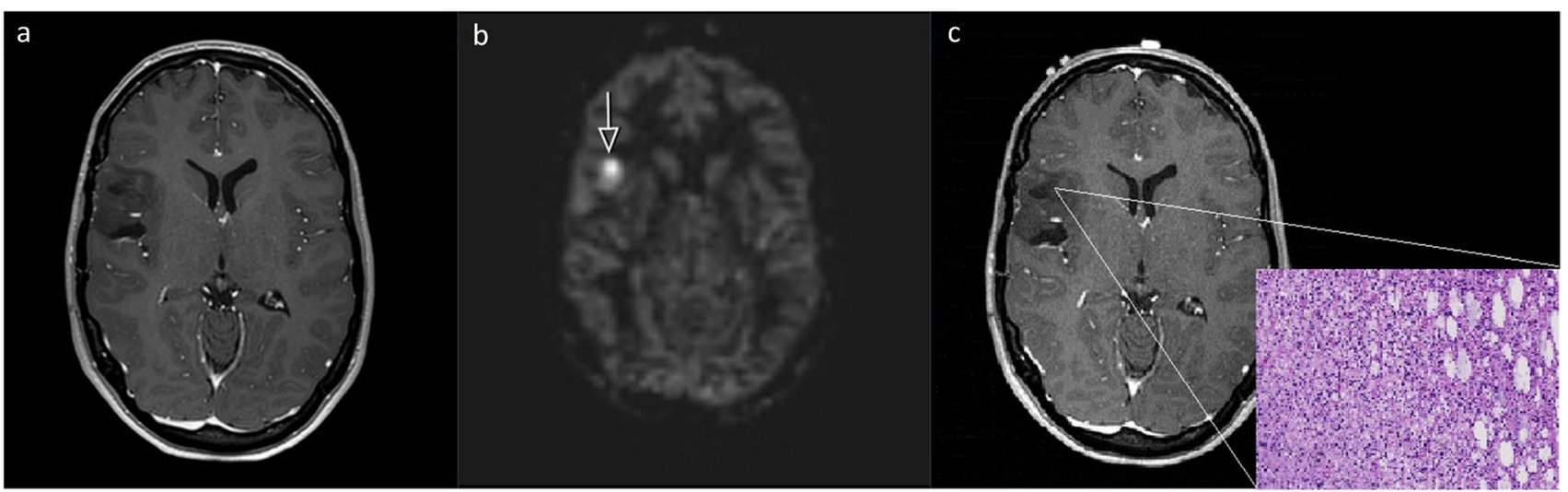

Fig. 2 Twenty-three-year-old woman with an untreated diffuse IDH mutated astrocytoma. False-positive scan; 2.a Index T1-MRI with contrast agent: no contrast enhancement. 2.b Index ASL rated as positive. 2.c Follow-up: pre-operative T1-MRI with contrast agent
(70 days after index MRI), no contrast enhancement, histology of recurrence resection $(\mathrm{H} \& \mathrm{E}$ staining, $\times 200)$ shows a low-grade lesion. All axial planes 


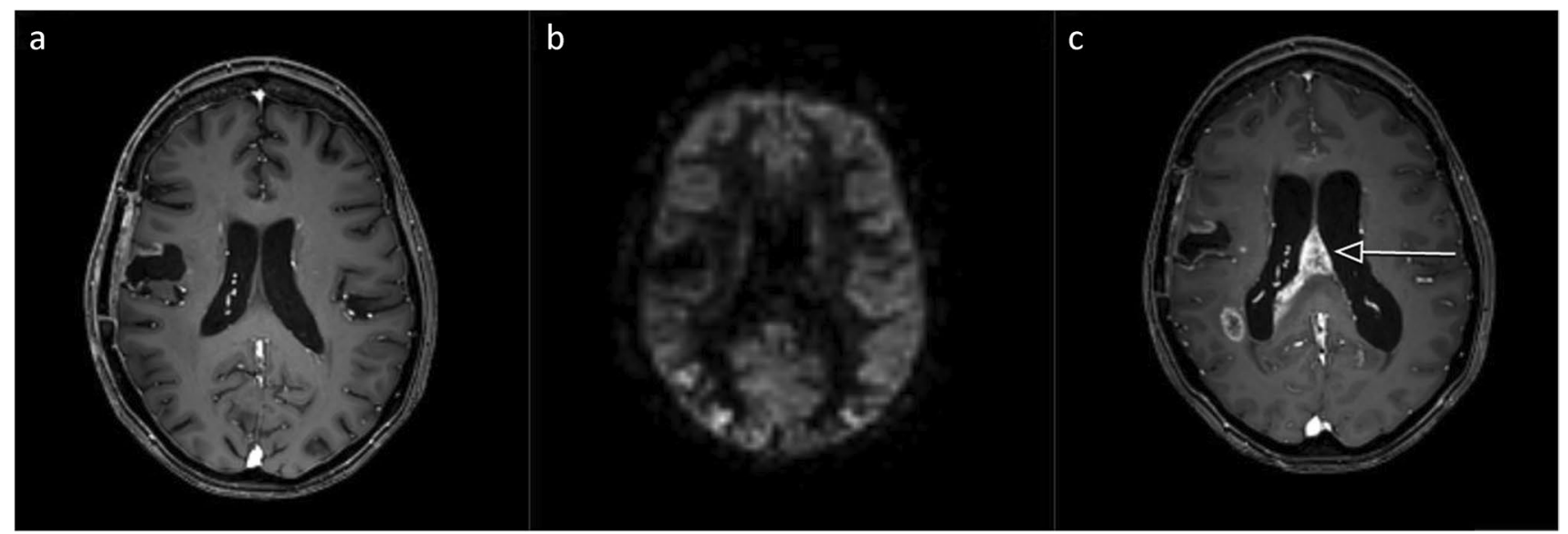

Fig. 3 Fifty-five-year-old woman with a diffuse IDH wildtype astrocytoma treated with radiotherapy and temozolomide. False-negative scan; 3.a Index T1-MRI with contrast agent: no contrast enhance-

circumscribed LGG without uptake [17]. More recently, Bashir et al. described $40{ }^{18} \mathrm{~F}$-FET-PET sessions of 42 patients with LGG showing positive uptake, from which $58 \%$ developed MP compared to 1 of 7 in the FET-negative group [18]. ASL-MRI has the advantage over PET and rCBV that ASL can easily be incorporated in standard-ofcare follow-up with MRI and does not require the use of a contrast agent.

\section{Limitations and strengths}

Firstly, the small sample size is a result of the recent implementation of ASL-MRIs in daily clinical practice ment. 3.b Index ASL rated as negative. 3.c Follow-up T1-MRI with contrast agent (203 days after index MRI): new contrast enhancement. All axial planes

and the low incidence of MP in LGG. Secondly, patients with the rare $1 \mathrm{p} / 19 \mathrm{q}$-codeleted glioma subtype (oligodendroglioma) were scarce. Consequently, the generalisability to the entire LGG population needs to be confirmed in a larger multi-centre consecutive cohort study. Thirdly, previous studies have found higher rCBV in grade II oligodendrogliomas compared to astrocytomas. This may also have influenced our analysis [19,20]. In our study, the six false-positively rated ASL-MRIs included one of the three oligodendroglioma controls and five of the 30 astrocytoma controls. Fourthly, we included patients with potential radiation necrosis/pseudoprogression, which may be mistaken for MP. However,

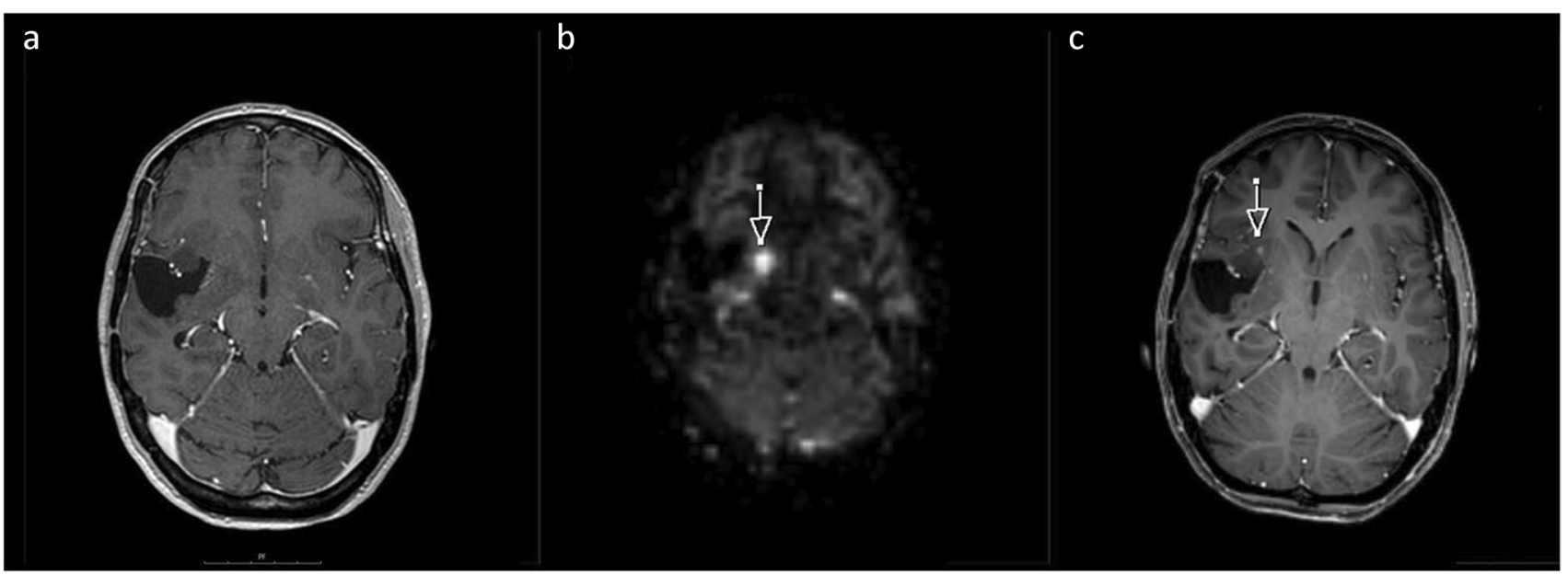

Fig. 4 Forty-three-year-old woman with an untreated oligodendroglioma. True positive scan; 4.a Index T1-MRI with contrast agent: no contrast enhancement. 4.b Index ASL rated as positive. 4.c Follow-up
T1-MRI with contrast agent (17 days after index MRI): new contrast enhancement. All axial planes 
such misinterpretation should be minimised by the long follow-up period. Fifthly, patients with no ASL-MRI available were excluded, which excluded all patients developing MP before 2016 and with follow-up imaging at another hospital. This could have resulted in a selection bias with more high-risk cases being followed in a university hospital. However, ASL imaging is included routinely in the follow-up of patients with LGG, and therefore, no patients being followed in this hospital after 2016 were excluded. Sixthly, not every progressive glioma shows contrast enhancement, and some patients may not have been recognised having MP in the case of no available histological outcome. However, histological confirmation is not always clinically feasible.

Lastly, ASL-MRI in itself represents a technique with potential for improvement with a relatively low spatial resolution and no defined widely approved quantitative perfusion threshold values. Therefore, we used a visual assessment method in this study, which was judged to be easier to implement in daily clinical practice and without the need for postprocessing tools.

Strengths of the study constituted the double, independent radiological assessment blinded to the clinical data, as well as the consideration of peri-ictal pseudoprogression. Furthermore, the prespecified assessment criteria can be named resulting in a substantial interrater agreement. Patients' tumour types were evaluated according to WHO 2016 criteria, and our results appeared to be independent of IDH-mutational status. To our knowledge, this is the largest study to date to examine the relation between ASL and subsequent MP in LGG.

Future studies are needed to validate our findings, study the specific value of ASL-MRI in the different tumour subtypes and to evaluate the added value of a quantitative analysis.

\section{Conclusion}

Perfusion imaging with ASL-MRI can detect malignant progression before T1-MRI within 12 months in treated and untreated patients with low-grade glioma with a sensitivity of $73 \%$ and a specificity of $82 \%$. A positive ASL-MRI during follow-up should therefore be a warning sign and encourage closer follow-up in these patients.

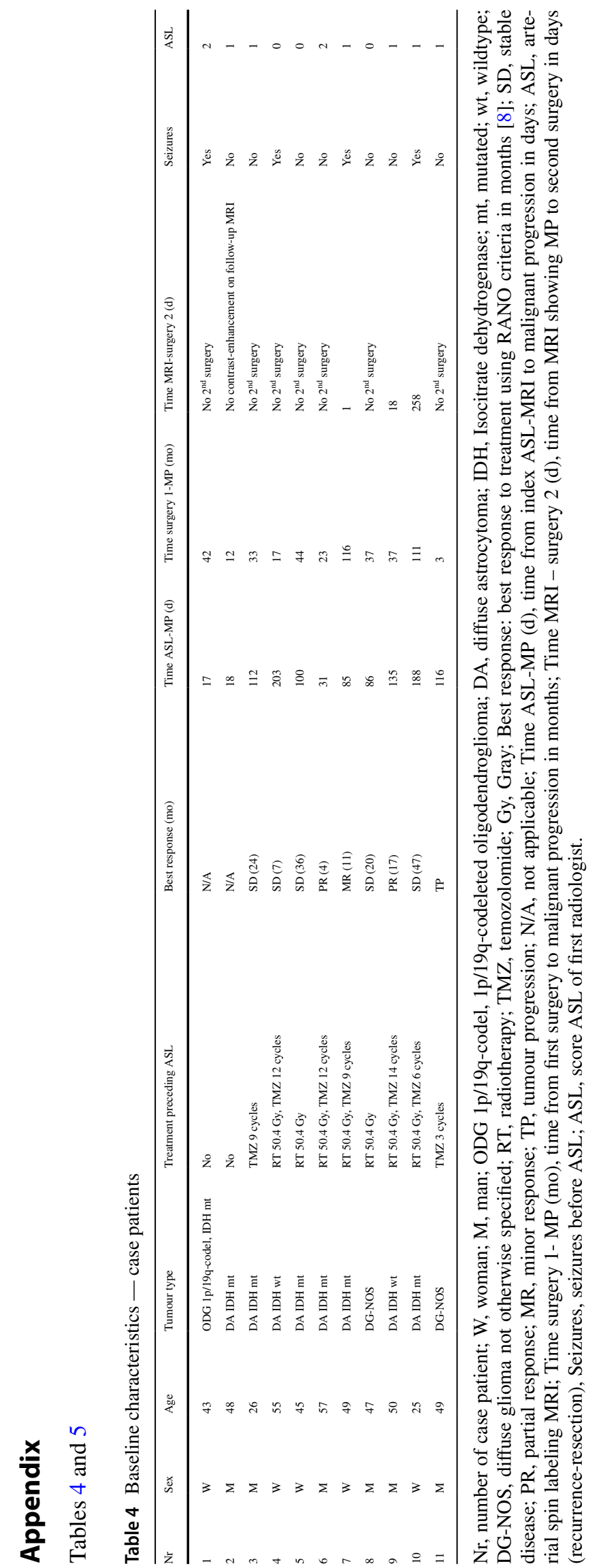


Table 5 Baseline characteristics — control patients

\begin{tabular}{|c|c|c|c|c|c|c|}
\hline $\mathrm{Nr}$ & Sex & Age & Tumour type & Treatment preceding ASL & $\begin{array}{l}\text { Seizures pre- } \\
\text { ceding ASL }\end{array}$ & Score ASL \\
\hline 1 & $\mathrm{~W}$ & 43 & ODG $1 \mathrm{p} / 19 \mathrm{q}$, codel & No & Yes & 1 \\
\hline 2 & $\mathrm{~W}$ & 49 & ODG $1 \mathrm{p} / 19 \mathrm{q}$, codel & No & No & 0 \\
\hline 3 & M & 47 & ODG $1 \mathrm{p} / 19 \mathrm{q}$, codel & No & No & 0 \\
\hline 4 & M & 48 & DA IDH mt & No & Yes & 0 \\
\hline 5 & M & 50 & DA IDH mt & No & Yes & 0 \\
\hline 6 & M & 40 & DG-NOS & No & No & 0 \\
\hline 7 & M & 26 & DA IDH mt & TMZ 9 cycles & No & 1 \\
\hline 8 & $\mathrm{~W}$ & 52 & DA IDH mt & No & No & 0 \\
\hline 9 & $\mathrm{~W}$ & 38 & DA IDH mt & No & No & 0 \\
\hline 10 & M & 25 & DA IDH mt & RT 50.4 Gy, TMZ 12 cycles & No & 0 \\
\hline 11 & $\mathrm{~W}$ & 44 & DA IDH mt & RT 50.4 Gy, TMZ 11 cycles & No & 0 \\
\hline 12 & M & 57 & DA IDH mt & RT 50.4 Gy, TMZ 12 cycles & No & 0 \\
\hline 13 & $\mathrm{~W}$ & 45 & DA IDH mt & RT 50.4 Gy & No & 0 \\
\hline 14 & M & 21 & DA IDH mt & RT 50.4 Gy & No & 0 \\
\hline 15 & $\mathrm{~W}$ & 21 & DA IDH mt & RT 50.4 Gy & No & 0 \\
\hline 16 & M & 57 & DA IDH mt & RT 50.4 Gy, TMZ 12 cycles & Yes & 2 \\
\hline 17 & M & 39 & DA IDH mt & RT 50.4 Gy, TMZ 12 cycles & No & 0 \\
\hline 18 & M & 18 & DA IDH mt & RT 50.4 Gy, TMZ 12 cycles & No & 0 \\
\hline 19 & W & 49 & DA IDH mt & RT 50.4 Gy, TMZ 9 cycles & No & 0 \\
\hline 20 & M & 25 & DA IDH mt & RT 50.4 Gy, TMZ 3 cycles & No & 0 \\
\hline 21 & M & 33 & DA IDH mt & RT 50.4 Gy, TMZ 2 cycles & No & 0 \\
\hline 22 & M & 53 & DA IDH mt & RT 50.4 Gy & No & 1 \\
\hline 23 & M & 32 & DG-NOS & RT 50.4 Gy & No & 0 \\
\hline 24 & $\mathrm{~W}$ & 27 & DG-NOS & RT 50.4 Gy & No & 0 \\
\hline 25 & M & 50 & DA IDH wt & RT 50.4 Gy, TMZ 8 cycles & No & 0 \\
\hline 26 & M & 31 & DA IDH mt & RT 50.4 Gy & No & 0 \\
\hline 27 & W & 50 & DG-NOS & RT 50.4 Gy, PCV 5 cycles & No & 0 \\
\hline 28 & M & 55 & DA IDH wt & RT 50.4 Gy, TMZ 7 cycles & No & 0 \\
\hline 29 & M & 46 & DA IDH mt & RT 50.4 Gy, TMZ 7 cycles & No & 0 \\
\hline 30 & M & 26 & DA IDH mt & RT 50.4 Gy, TMZ 6 cycles & No & 2 \\
\hline 31 & M & 45 & DA IDH wt & No & Yes & 0 \\
\hline 32 & M & 35 & DA IDH mt & No & No & 0 \\
\hline 33 & W & 23 & DA IDH mt & No & Yes & 2 \\
\hline
\end{tabular}

$\mathrm{Nr}$, number of control patient; W, woman; M, man; Age, age at first diagnosis; ODG 1p/19q-codel, 1p/19qcodeleted oligodendroglioma; DA, diffuse astrocytoma; IDH, Isocitrate dehydrogenase; mt, mutated; wt, wildtype; DG-NOS, diffuse glioma not otherwise specified; ASL, arterial spin labeling MRI; Score ASL, of first radiologist; RT, radiotherapy; Gy, Gray; TMZ, temozolomide; PCV, procarbazine/lomustine/vincristine.
Acknowledgements We wish to acknowledge Angelika Muhlebner for providing the histological image for Fig. 2c.

Author contribution All authors contributed to the study conception and design. Material preparation, data collection and analysis were performed by Christina M. Flies, Tom J. Snijders, Jan Willem Dankbaar and Tom van Seeters. The first draft of the manuscript was written by Christina M. Flies and Tom J. Snijders, and all authors commented on previous versions of the manuscript. All authors read and approved the final manuscript.

Funding This work was supported by a donation of the Stophersenkanker.nu foundation to TJS and FYFdV and by Foundation Vrienden UMC Utrecht.
Data availability Individual, anonymised data is included in the Appendix.

Code availability Not applicable.

\section{Declarations}

Conflict of interest The authors have no relevant financial or non-financial interests to disclose.

Ethics approval This retrospective chart review study involving human participants was in accordance with the ethical standards of the insti- 
tutional and national research committee and with the 1964 Helsinki Declaration and its later amendments or comparable ethical standards. The institutional Medical Ethical/Biobank Committee of the University Medical Center Utrecht approved the use of patient data in the context of an overlapping not yet published study in progress, for which patients had provided written informed consent (protocol no. 16-342/16-340).

Consent to participate Informed consent was obtained from all individual participants included in the study.

Consent for publication Additional informed consent was obtained from all individual participants for whom identifying information is included in this article.

Open Access This article is licensed under a Creative Commons Attribution 4.0 International License, which permits use, sharing, adaptation, distribution and reproduction in any medium or format, as long as you give appropriate credit to the original author(s) and the source, provide a link to the Creative Commons licence, and indicate if changes were made. The images or other third party material in this article are included in the article's Creative Commons licence, unless indicated otherwise in a credit line to the material. If material is not included in the article's Creative Commons licence and your intended use is not permitted by statutory regulation or exceeds the permitted use, you will need to obtain permission directly from the copyright holder. To view a copy of this licence, visit http://creativecommons.org/licenses/by/4.0/.

\section{References}

1. Ostrom QT, Cioffi G, Gittleman H, Patil N, Waite K, Kruchko C, Barnholtz-Sloan JS (2019) CBTRUS statistical report: primary brain and other central nervous system tumors diagnosed in the United States in 2012-2016. Neuro Oncol 21(Supplement_5):v1v100. https://doi.org/10.1093/neuonc/noz150

2. Louis DN, Perry A, Reifenberger G, von Deimling A, FigarellaBranger D, Cavenee WK, Ohgaki H, Wiestler OD, Kleihues P, Ellison DW (2016) The 2016 World Health Organization Classification of Tumors of the Central Nervous System: a summary. Acta Neuropathol 131(6):803-820. https://doi.org/10.1007/ s00401-016-1545-1

3. Buckner JC, Shaw EG, Pugh SL, Chakravarti A, Gilbert MR, Barger GR, Coons S, Ricci P, Bullard D, Brown PD, Stelzer K, Brachman D, Suh JH, Schultz CJ, Bahary JP, Fisher BJ, Kim H, Murtha AD, Bell EH, Won M, Mehta MP, Curran WJ Jr (2016) Radiation plus procarbazine, $\mathrm{CCNU}$, and vincristine in low-grade glioma. N Engl J Med 374(14):1344-55. https://doi.org/10.1056/ NEJMoa1500925

4. Claus EB, Walsh KM, Wiencke JK, Molinaro AM, Wiemels JL, Schildkraut JM, Bondy ML, Berger M, Jenkins R, Wrensch M (2015) Survival and low-grade glioma: the emergence of genetic information. Neurosurg Focus 38(1):E6. https://doi.org/10.3171/ 2014.10.FOCUS12367

5. Smith JS, Chang EF, Lamborn KR, Chang SM, Prados MD et al (2008) Role of extent of resection in the long-term outcome of low-grade hemispheric gliomas. J Clin Oncol 26(8):1338-1345. https://doi.org/10.1200/JCO.2007.13.9337

6. Murphy ES, Leyrer CM, Parsons M, Suh JH, Chao ST, Yu JS, Kotecha R, Jia X, Peereboom DM, Prayson RA, Stevens GHJ,
Barnett GH, Vogelbaum MA, Ahluwalia MS (2018) Risk factors for malignant transformation of low-grade glioma. Int J Radiat Oncol Biol Phys 100(4):965-971. https://doi.org/10.1016/j.jjrobp. 2017.12.258

7. Weller M, van den Bent M, Tonn JC, Stupp R, Preusser M, CohenJonathan-Moyal E, Henriksson R, LeRhun E, Balana C, Chinot O, Bendszus M, Reijneveld JC, Dhermain F, French P, Marosi C, Watts C, Oberg I, Pilkington G, Baumert BG, Taphoorn MJB, Hegi M, Westphal M, Reifenberger G, Soffietti R, Wick W (2017) European Association for Neuro-Oncology (EANO) guideline on the diagnosis and treatment of adult astrocytic and oligodendroglial gliomas. Lancet Oncol 18(6):e315-e329. https://doi.org/10. 1016/s1470-2045(17)30194-8

8. Wen PY, Macdonald DR, Reardon DA, Cloughesy TF, Sorensen AG et al (2010) Updated response assessment criteria for highgrade gliomas: response assessment in neuro-oncology working group. J Clin Oncol 28(11):1963-1972. https://doi.org/10.1200/ JCO.2009.26.3541

9. Thust SC, Heiland S, Falini A, Jager HR, Waldman AD, Sundgren PC, Godi C, Katsaros VK, Ramos A, Bargallo N, Vernooij MW, Yousry T, Bendszus M, Smits M (2018) Glioma imaging in Europe: a survey of 220 centres and recommendations for best clinical practice. Eur Radiol 28(8):3306-3317. https://doi.org/10. 1007/s00330-018-5314-5

10. Danchaivijitr N, Waldman AD, Tozer DJ, Benton CE, Caseiras GB, Tofts PS, Rees JH, Jäger HR (2008) Low-grade gliomas: do changes in rCBV measurements at longitudinal perfusionweighted MR imaging predict malignant transformation? Radiology 247(1):170-178. https://doi.org/10.1148/radiol.2471062089

11. Petcharunpaisan S, Ramalho J, Castillo M (2010) Arterial spin labeling in neuroimaging. World J Radiol 2(10):384-398. https:// doi.org/10.4329/wjr.v2.i10.384

12. Grade M, Hernandez Tamames JA, Pizzini FB, Achten E, Golay $X$ et al (2015) A neuroradiologist's guide to arterial spin labeling MRI in clinical practice. Neuroradiology 57(12):1181-1202. https://doi.org/10.1007/s00234-015-1571-z

13. Morana G, Piccardo A, Tortora D, Puntoni M, Severino M, Nozza P, Ravegnani M, Consales A, Mascelli S, Raso A, Cabria M, Verrico A, Milanaccio C, Rossi A (2017) Grading and outcome prediction of pediatric diffuse astrocytic tumors with diffusion and arterial spin labeling perfusion MRI in comparison with 18F-DOPA PET. Eur J Nucl Med Mol Imaging 44(12):20842093. https://doi.org/10.1007/s00259-017-3777-2

14. Rheims S, Ricard D, van den Bent M, Taillandier L, Bourg V, Desestret V, Cartalat-Carel S, Hermier M, Monjour A, Delattre JY, Sanson M, Honnorat J, Ducray F (2011) Peri-ictal pseudoprogression in patients with brain tumor. Neuro Oncol 13(7):775782. https://doi.org/10.1093/neuonc/nor082

15. Sim J, Wright CC (2005) The kappa statistic in reliability studies: use, interpretation, and sample size requirements. Phys Ther 85(3):257-268. https://doi.org/10.1093/ptj/85.3.257

16. Chen IE, Swinburne N, Tsankova NM, Hefti MM, Aggarwal A, Doshi AH, Hormigo A, Delman BN, Nael K (2018) Sequential apparent diffusion coefficient for assessment of tumor progression in patients with low-grade glioma. AJNR Am J Neuroradiol 39(6):1039-1046. https://doi.org/10.3174/ajnr.A5639

17. Floeth FW, Pauleit D, Sabel M, Stoffels G, Reifenberger G, Riemenschneider MJ, Jansen P, Coenen HH, Steiger HJ, Langen KJ (2007) Prognostic value of O-(2-18F-fluoroethyl)-ltyrosine PET and MRI in low-grade glioma. J Nucl Med 48(4):519-527. https:// doi.org/10.2967/jnumed.106.037895 
18. Bashir A, Brennum J, Law I (2019) The diagnostic accuracy of detecting malignant transformation of low-grade glioma using O-(2-[18F]fluoroethyl)-1-tyrosine positron emission tomography: a retrospective study. J Neurosurg 130:451-464. https://doi.org/ 10.3171/2017.8.JNS171577

19. Cha S, Tihan T, Crawford F, Fischbein NJ, Chang S, Bollen A, Nelson SJ, Prados M, Berger MS, Dillon WP (2005) Differentiation of low-grade oligodendrogliomas from low-grade astrocytomas by using quantitative blood-volume measurements derived from dynamic susceptibility contrast-enhanced MR imaging. Am J Neuroradiol 26(2):266-273
20. Lev MH, Ozsunar Y, Henson JW, Rasheed AA, Barest GD, Harsh GR IV, Fitzek MM, Chiocca EA, Rabinov JD, Csavoy AN, Rosen BR, Hochberg FH, Schaefer PW, Gilberto Gonzalez R (2004) Glial tumor grading and outcome prediction using dynamic spinecho MR susceptibility mapping compared with conventional contrast-enhanced MR: confounding effect of elevated rCBV of oligodendroglimoas. Am J Neuroradiol 25(2):214-221

Publisher's note Springer Nature remains neutral with regard to jurisdictional claims in published maps and institutional affiliations. 